\title{
Gamma-ray binaries and recent results on LS I +61303
}

\author{
X. Paredes-Fortuny, ${ }^{* 1}$, M. Ribó ${ }^{1,}$, V. Bosch-Ramon ${ }^{1}$, J. Casares ${ }^{2,3}$, D. del Ser \\ Badia $^{1,4}$, O. Fors ${ }^{5,1}$, and J. Núñez ${ }^{1,4}$
}

${ }^{1}$ Dept. d'Astronomia i Meteorologia, Institut de Ciènces del Cosmos (ICCUB), Universitat de Barcelona (IEEC-UB), Martí i Franquès 1, E08028 Barcelona, Spain

${ }^{2}$ Instituto de Astrofísica de Canarias, E-38205 La Laguna, Santa Cruz de Tenerife, Spain

${ }^{3}$ Departamento de Astrofísica, Universidad de La Laguna, E-38206 La Laguna, Santa Cruz de Tenerife, Spain

${ }^{4}$ Observatori Fabra, Reial Acadèmia de Ciències i Arts de Barcelona, Rambla dels Estudis, 115, 08002 Barcelona, Spain

${ }^{5}$ Department of Physics and Astronomy, University of North Carolina at Chapel Hill, Chapel Hill, NC 27599-3255, USA

E-mail: xparedes Cam.ub.es

Gamma-ray binaries are binary systems hosting an early type star and a compact object, either a black hole or a neutron star, with the non-thermal flux peaking at gamma-rays. Currently we know five gamma-ray binaries, all of them have the X-ray and gamma-ray flux modulated with the orbital period, and present a diverse set of orbital parameters. The nature of the compact object is only know for one of them, which is a pulsar, but probably the rest of gamma-ray binaries host a pulsar as well. In this work we present optical photometric and $E W_{\mathrm{H} \alpha}$ results on the gamma-ray binary LS I +61 303. This system hosts a Be star with a circumstellar decretion disk surrounding the star, therefore we expect variability in the thermal flux due to the disruption of the circumstellar disk by the compact object tidal forces and the putative pulsar wind close to the periastron passage, every 26.5 days. This binary system also exhibits a superorbital variability of 4.6 years in the radio/X-ray (non-thermal indicators) flux and phase of the maximum and in the $E W_{\mathrm{H} \alpha}$ flux, related to periodic changes in the circumstellar disk. We detect a positive orbital phase shift and flux increase in the optical photometry and $E W_{\mathrm{H} \alpha}$ (tracers of thermal emission), showing that there is a strong coupling between the thermal and non-thermal emission processes in the gamma-ray binary LS I +61 303. In addition, the optical photometry presents a lag of $\sim 0.1-0.2$ orbital phases with respect the $E W_{\mathrm{H} \alpha}$, explained considering different emitting regions.

XI Multifrequency Behaviour of High Energy Cosmic Sources Workshop

25-30 May 2015

Palermo, Italy

\footnotetext{
*Speaker.

${ }^{\dagger}$ Serra Húnter Fellow.
} 


\section{Gamma-ray binaries}

Gamma-ray binaries are systems that comprise a compact object orbiting a companion star that have the non-thermal maximum of the Spectral Energy Distribution in gamma-rays. Currently we know five gamma-ray binaries: PSR B1259-63, LS I +61 303, LS 5039, HESS J0632+057, and 1FGL J1018.6-5856. All of them host an early type star and a compact object of unknown nature, either a black hole or a neutron star, except for the binary system PSR B1259-63 where pulsations have already been detected. VLBI observations show extended, and elongated, cometary tail-like morphologies, hinting that the compact object might be a pulsar. LS 5039 and 1FGL J1018.6-5856 host an O6 star, while LS I +61 303, HESS J0632, and PSR B1259-63 host a Be star (Be stars are non-supergiant fast-rotating early type star which at some points have shown emission lines). The five gamma-ray binaries are located at a distance between 1.5 and $5 \mathrm{kpc}$. The orbital period ranges from 4 days for the binary LS 5039 up to 3.5 years for the system PSR B1259-63, and the eccentricity ranges from 0.35 until 0.87 , respectively, resulting in very different periastronapastron distances. X-ray and TeV emission is modulated with the orbital period. See $[1,2,3]$ and references therein.

Currently, there are two proposed physical scenarios that explain the gamma-ray emission from gamma-ray binaries: the microquasar scenario and the pulsar wind scenario [4]. In both scenarios we find the proper environment for particle acceleration (magnetized plasmas, high density contrast, relativistic motion, shocks, and turbulence), and non-thermal processes (synchrotron and inverse Compton for electrons, and proton-proton interactions) [5, 1]. The thermal emission in the Gamma-ray binaries hosting a Be star is expected to come from the optical star and its circumstellar decretion disk (Be stars are fast spinning stars) [6]. SPH simulations [7, 8] suggest that the stellar decretion disk is perturbed/disrupted during the periastron passage of the compact object by the tidal forces and or the putative pulsar wind.

\section{Optical results on the gamma-ray binary LS I +61 303}

LS I +61 303 is a gamma-ray binary [1,2] composed of an optical star with spectral type B0 Ve [9] and magnitude $V \sim 10.7$ [10], and a compact companion orbiting in a highly eccentric orbit, $e=0.54-0.72[11,6]$, with a period of $26.496 \mathrm{~d}$ [12]. The source is located at a distance of $\sim 2 \mathrm{kpc}$ [13]. Modulated emission with the orbital period has been detected in radio [14], optical photometry [15], $\mathrm{H} \alpha$ spectroscopy [16], X-rays [17], GeV [18], and TeV [19]. LS I +61 303 also shows a $\sim 4.6 \mathrm{yr}$ superorbital modulation in radio flux and phase of the maximum [12], and in the $\mathrm{H} \alpha$ equivalent width $\left(E W_{\mathrm{H} \alpha}\right)$ flux [20], suggesting that the superorbital variability might be related to periodic changes in the circumstellar disk [21]. This superorbital modulation has also been observed in X-rays [22, 23], and in High Energy gamma-rays $(E>100 \mathrm{MeV})$ [24]. The periastron passage occurs at orbital phase $0.23-0.28[6,11]$ (with zero orbital phase set at JD 2443 366.775 [12]).

In this work, we present contemporaneous optical photometric and $E W_{\mathrm{H} \alpha}$ observations of LS I +61 303 spanning 1.5 yr. Preliminary results have been presented in [25]. 


\subsection{Observations and data reduction}

The optical photometric observations of LS I +61 303 have been conducted with the Telescope Fabra-ROA Montsec (TFRM) installed at the Observatori Astronòmic del Montsec (Lleida, Spain). The TFRM has an aperture of $0.5 \mathrm{~m}$, a refurbished Backer-Nunn Camera, a focal ratio $f / 0.96$, a field of view of $4.4^{\circ} \times 4.4^{\circ}$, a passband filter $\lambda>475 \mathrm{~nm}$, and a CCD based on FLI ProLine 16803 with quantum efficiency of $60 \%$ at $550 \mathrm{~nm}$ [26]. The observations span from 2012 July 31 to 2014 March 7 (71 nights of good data), including two observational campaigns centered at Autumn 2013 and 2014, respectively (hereafter Season 1 and Season 2). A standard calibration has been conducted and the light-curves have been corrected using differential photometry. Details on the data reduction can be found in [27].

Contemporaneous $E W_{\mathrm{H} \alpha}$ observations of LS I +61 303 have been obtained using FRODOspec on the robotic $2.0 \mathrm{~m}$ Liverpool telescope installed at the Observatorio del Roque de los Muchcachos (La Palma, Spain). The observations span from 2012 July 2 to 2014 January 15 (104 nights of good data). Details on the data reduction can be found in [28].

\subsection{Results}

The optical photometric and $E W_{\mathrm{H} \alpha}$ light-curves of the LS I +61 303 folded with the orbital period of 26.5 d [12], and their sinusoidal fits for the Season 1 and 2 are shown in Fig. 1. The sinusoidal fits reveal a positive orbital phase shift between the two observational campaigns of $0.16 \pm 0.03$ at $5.2 \sigma$ confidence level (c.l.) for the optical photometry and a phase shift of $0.22 \pm$ 0.05 at $4.2 \sigma$ c.l. for the $E W_{\mathrm{H} \alpha}$. This result indicates that the optical photometry and the $E W_{\mathrm{H} \alpha}$ (thermal emission) exhibit a superorbital variability, as that found in radio and X-rays (non-thermal emission).

A color map of the optical photometry and $E W_{\mathrm{H} \alpha}$ flux as a function of the orbital phase and superorbital cycle, including the X-ray and radio flux trends [23] is shown in Fig. 2. For a given superorbital phase, the optical flux shows a lag of $0.1-0.2$ in orbital phases with respect the $E W_{\mathrm{H} \alpha}$ flux. In addition, the optical flux shows a lag of $\sim 0.1$ and $\sim 0.3$ orbital phases with respect the radio and X-ray outburst at equivalent superorbital phases one superorbital cycle a part [23].

\subsection{Discussion}

The circumstellar decretion disk is likely to be perturbed by the compact object tidal forces and the pulsar wind ram pressure (if the compact object is a pulsar), being the maximum perturbation close to the periastron passage at phase $\phi_{\text {per }}=0.23$. Figure 2 allows us to extract the chronological order of the maximum flux emission at different wavelengths. For a given superorbital phase, after periastron passage at orbital phase $\phi_{\mathrm{per}}=0.23$, first there is a maximum in X-rays (tracer of nonthermal emission), it is followed by a maximum in $E W_{\mathrm{H} \alpha}$ (tracer of the outer disk conditions) and in radio emission (which is produced mainly outside the binary system). Finally a maximum in the optical photometry is observed (which traces the variability from the inner circumstellar disk).

The optical flux shows $\sim 0.06$ mag modulation (first observational campaign), which corresponds to $\sim 6 \%$ in the total flux. The Be disk represents the $35 \%$ of the flux [6], therefore we observe a $\sim 16 \%$ variability in the disk flux. On the other hand, $E W_{\mathrm{H} \alpha}$ exhibits a $\sim 25 \%$ variability (first observational campaign), this implies that the external parts of the disk are more perturbed 

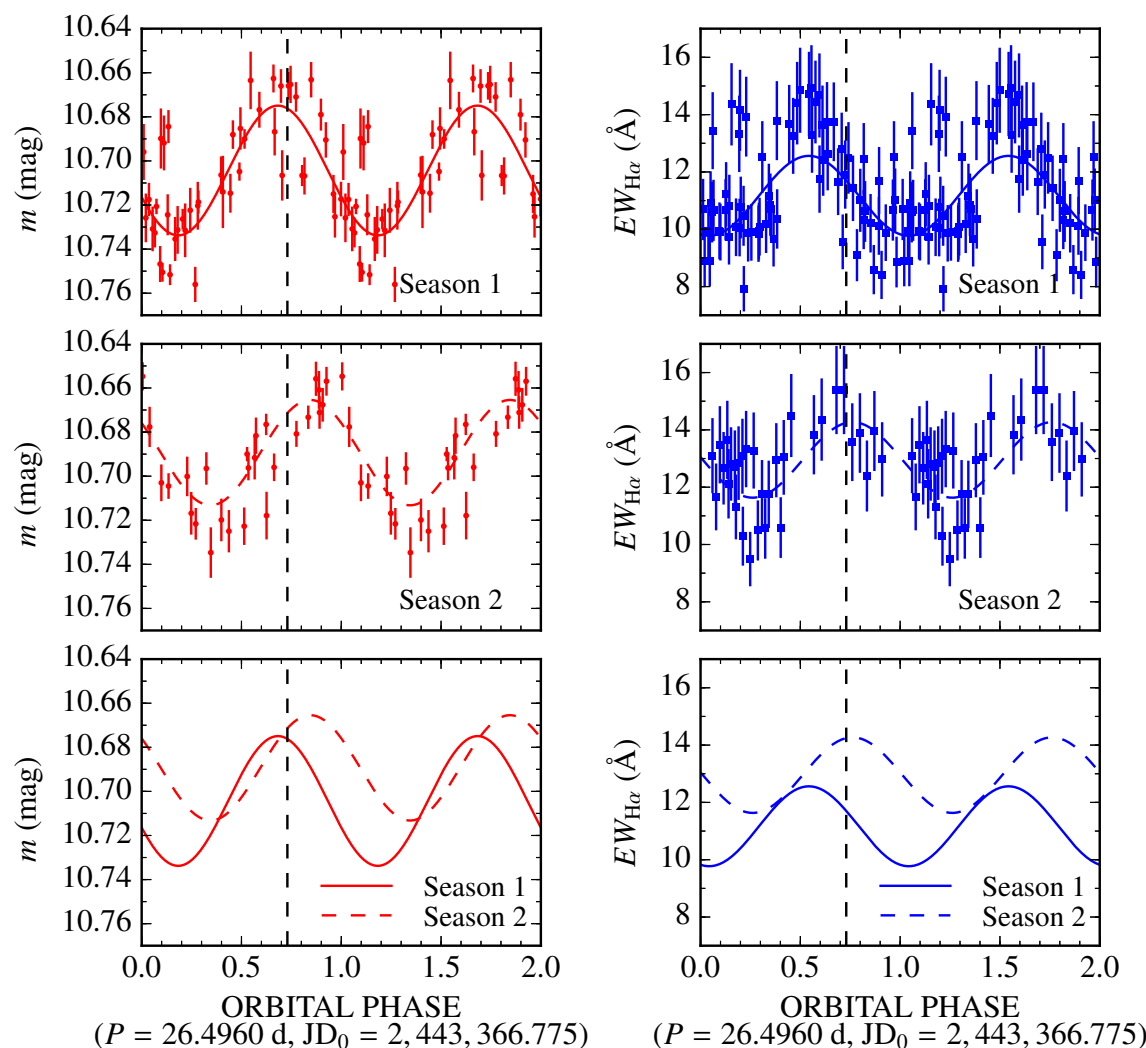

Figure 1: Optical photometric (left) and $E W_{\mathrm{H} \alpha}$ (right) light-curves folded with the orbital period and their sinusoidal fits to the orbital variability. Error bars represent $1 \sigma$ uncertainties. Two cycles are displayed for clarity. Adapted from [27].

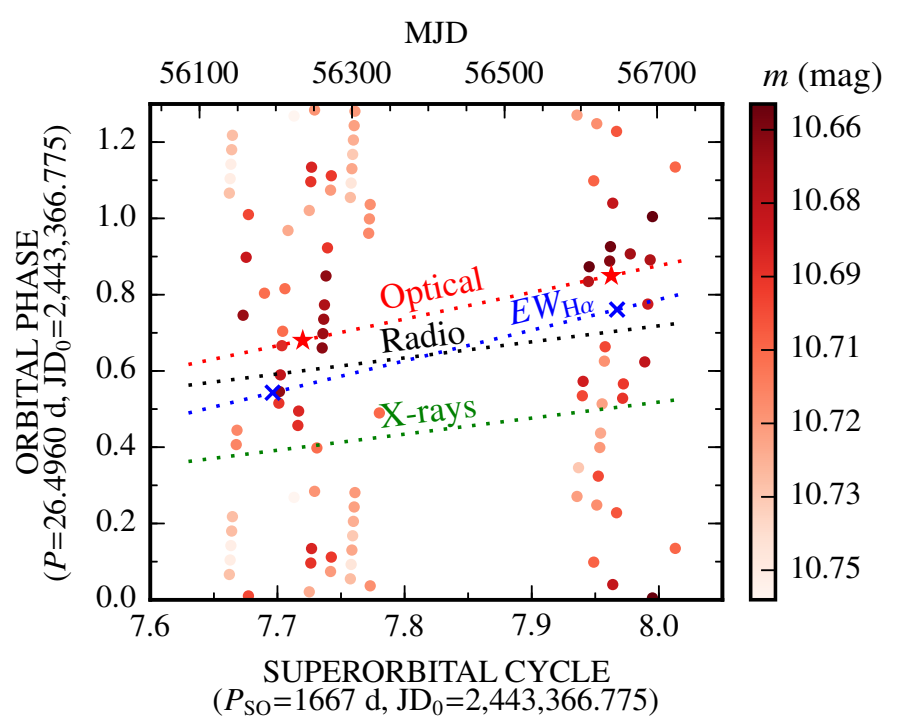

Figure 2: Color map of the optical photometry as a function of the orbital phase and superorbital cycle. The position of the red stars and blue crosses is obtained from the sinusoidal fits of each observational campaign, for the optical photometry and $E W_{\mathrm{H} \alpha}$, respectively. The radio and X-ray trends are taken from [23] and correspond to the previous superorbital cycle. Adapted from [27]. 
than the internal ones. Furthermore, the $\sim 0.1$ orbital phase lag between the optical photometry and the $E W_{\mathrm{H} \alpha}$, suggests that the external parts are perturbed before the internal parts (and also recover before the internal parts).

The $E W_{\mathrm{H} \alpha}$ superorbital variability has been associated with periodic changes on the Be star and/or its circumstellar disk [29], e.g., the presence of a moving one-armed spiral density wave in the disk [21]. We have detected an orbital phase drift in the optical (thermal) as that observed in radio and X-rays (non-thermal), therefore we have shown that there is an empirical coupling between the thermal and non-thermal emission in the gamma-ray binary LS I +61 303 .

\section{Questions \& Answers}

Q: Is there any optical spectropolarimetric measurement of LS I +61 303? In a system with a high eccentric orbit plus a periodic disruption of the accretion disk, important variations are expected in terms of polarization degree (linear polarization) and polarization position angle. (Question by Frédéric Marin)

A: There are two works $[30,31]$ on spectropolarimetry of LS I +61 303 but a systematic monitoring has not been conducted yet.

\section{References}

[1] Dubus, G. 2013, A\&A Rev., 21, 64

[2] Paredes, J. M., Bednarek, W., Bordas, P., et al. 2013, Astroparticle Physics, 43, 301

[3] Porter, J. M., \& Rivinius, T. 2003, PASP, 115, 1153

[4] Mirabel, I. F. 2012, Science, 335, 175

[5] Bosch-Ramon, V., \& Khangulyan, D. 2009, International Journal of Modern Physics D, 18, 347

[6] Casares, J., Ribas, I., Paredes, J. M., Martí, J., \& Allende Prieto, C. 2005, MNRAS, 360, 1105

[7] Takata, J., Okazaki, A. T., Nagataki, S., et al. 2012, ApJ, 750, 70

[8] Okazaki, A. T., Nagataki, S., Naito, T., et al. 2011, PASJ, 63, 893

[9] Hutchings, J. B., \& Crampton, D. 1981, PASP, 93, 486

[10] Paredes, J. M., \& Figueras, F. 1986, A\&A, 154, L30

[11] Aragona, C., McSwain, M. V., Grundstrom, E. D., et al. 2009, ApJ, 698, 514

[12] Gregory, P. C. 2002, ApJ, 575, 427

[13] Frail, D. A., \& Hjellming, R. M. 1991, AJ, 101, 2126

[14] Taylor, A. R., \& Gregory, P. C. 1982, ApJ, 255, 210

[15] Mendelson, H., \& Mazeh, T. 1989, MNRAS, 239, 733

[16] Paredes, J. M., Marziani, P., Marti, J., et al. 1994, A\&A, 288, 519

[17] Paredes, J. M., Martí, J., Peracaula, M., \& Ribó, M. 1997, A\&A, 320, L25

[18] Abdo, A. A., Ackermann, M., Ajello, M., et al. 2009, ApJ, 701, L123 
[19] Albert, J., Aliu, E., Anderhub, H., et al. 2009, ApJ, 693, 303

[20] Zamanov, R., Stoyanov, K., Martí, J., et al. 2013, A\&A, 559, A87

[21] Negueruela, I., Reig, P., Coe, M. J., \& Fabregat, J. 1998, A\&A, 336, 251

[22] Li, J., Torres, D. F., Zhang, S., et al. 2012, ApJ, 744, L13

[23] Chernyakova, M., Neronov, A., Molkov, S., et al. 2012, ApJ, 747, L29

[24] Ackermann, M., Ajello, M., Ballet, J., et al. 2013, ApJ, 773, L35

[25] Paredes-Fortuny, X., Ribó, M., Fors, O., Núñez, J., \& Bosch-Ramon, V. 2014, International Journal of Modern Physics Conference Series, 28, 1460197

[26] Fors, O., Núñez, J., Luis Muiños, J., et al. 2013, PASP, 125, 522

[27] Paredes-Fortuny, X., Ribó, M., Bosch-Ramon, V., et al. 2015, A\&A, 575, L6

[28] Casares, J., Ribó, M., Ribas, I., et al. 2012, MNRAS, 421, 1103

[29] Zamanov, R. K., Martí, J., Paredes, J. M., et al. 1999, A\&A, 351, 543

[30] Nagae, O., Kawabata, K. S., Fukazawa, Y., et al. 2006, PASJ, 58, 1015

[31] Nagae, O., Kawabata, K. S., Fukazawa, Y., et al. 2009, AJ, 137, 3509

\section{Acknowledgments}

The authors acknowledge support of the TFRM team for preparing and carrying out the optical photometric observations. We acknowledge support by the Spanish Ministerio de Economía y Competitividad (MINECO) under grants AYA2013-47447-C3-1-P, AYA2010-18080, FPA201348381-C6-6-P, and MDM-2014-0369 of ICCUB (Unidad de Excelencia 'María de Maeztu'). This research has been supported by the Marie Curie Career Integration Grant 321520. X.P.-F. acknowledges financial support from Universitat de Barcelona and Generalitat de Catalunya under grants APIF and FI (2015FI_B1 00153), respectively. V.B-R. acknowledges financial support from MINECO and European Social Funds through a Ramón y Cajal fellowship. 